Louisiana State University

LSU Digital Commons

Faculty Publications

Department of Mathematics

$12-27-2016$

\title{
New prediction approach for stabilizing time-varying systems under time-varying input delay
}

Frederic Mazenc

Laboratoire des Signaux et Systèmes

Michael Malisoff

Louisiana State University

Follow this and additional works at: https://digitalcommons.Isu.edu/mathematics_pubs

\section{Recommended Citation}

Mazenc, F., \& Malisoff, M. (2016). New prediction approach for stabilizing time-varying systems under time-varying input delay. 2016 IEEE 55th Conference on Decision and Control, CDC 2016, 3178-3182. https://doi.org/10.1109/CDC.2016.7798746

This Conference Proceeding is brought to you for free and open access by the Department of Mathematics at LSU Digital Commons. It has been accepted for inclusion in Faculty Publications by an authorized administrator of LSU Digital Commons. For more information, please contact ir@lsu.edu. 


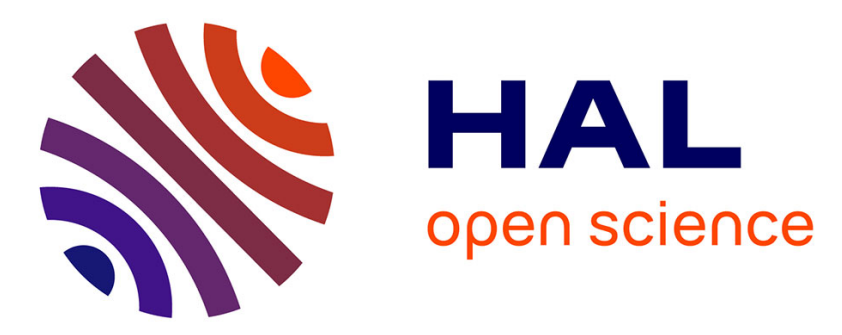

\section{New Prediction Approach for Stabilizing Time-Varying Systems under Time-Varying Input Delay}

Frédéric Mazenc, Michael Malisoff

\section{To cite this version:}

Frédéric Mazenc, Michael Malisoff. New Prediction Approach for Stabilizing Time-Varying Systems under Time-Varying Input Delay. 55th IEEE Conference on Decision and Control (CDC 2016), Dec 2016, Las Vegas, United States. 10.1109/cdc.2016.7798746 . hal-01389869v2

\section{HAL Id: hal-01389869 \\ https://hal.inria.fr/hal-01389869v2}

Submitted on 14 Jan 2017

HAL is a multi-disciplinary open access archive for the deposit and dissemination of scientific research documents, whether they are published or not. The documents may come from teaching and research institutions in France or abroad, or from public or private research centers.
L'archive ouverte pluridisciplinaire HAL, est destinée au dépôt et à la diffusion de documents scientifiques de niveau recherche, publiés ou non, émanant des établissements d'enseignement et de recherche français ou étrangers, des laboratoires publics ou privés. 


\section{New Prediction Approach for Stabilizing Time-Varying Systems under Time-Varying Input Delay}

\author{
Frédéric Mazenc
}

\author{
Michael Malisoff
}

\begin{abstract}
We provide a new sequential predictors approach for the exponential stabilization of linear time-varying systems with pointwise time-varying input delays. Our method circumvents the problem of constructing and estimating distributed terms in the stabilizing control laws, and allows arbitrarily large input delay bounds. We illustrate our method using a pendulum dynamics.
\end{abstract}

Key Words-Delay, robustness, stability, time-varying

\section{INTRODUCTION}

This work continues our search (which we began in [11]) for predictive methods for time-varying systems that can be applied without computing Lie derivatives and without computing distributed terms, and which compensate for arbitrarily long input delays. Our work is motivated by the ubiquity of input delays across engineering, and the challenges that can occur when building delay tolerant controls, if one applies traditional methods that have distributed terms. See, e.g., [4] and [17], and [6], [15], [18] for electromechanical input delays in muscle response in neuromuscular electrical stimulation (or NMES). For constant coefficient linear systems, it often suffices to use linear matrix inequalities (or LMIs) to build delay tolerant controls, but many important linear systems are time-varying. For instance, when tracking reference trajectories under input delays and linearizing around the reference trajectories, one obtains time-varying linear systems, even if the original system is time invariant.

Traditional input delay compensation methods can roughly be grouped into three categories of approaches. One approach is to solve the control problem with the input delay set to 0 , and to then look for upper bounds on the input delay that the resulting closed loop system can tolerate without sacrificing the desired stability properties. Two advantages of this socalled emulation approach are that (a) it makes it possible to use relatively simple controls for undelayed systems (such as Lie derivative feedback design methods and other approaches from [7]) and (b) the strict Lyapunov functions one often obtains from solving the feedback design problem for the corresponding undelayed system can often be transformed into Lyapunov-Krasovskii functionals, which can in turn be used to compute bounds on the input delays that the system can tolerate. See [12] for ways to transform strict Lyapunov

Mazenc is with EPI DISCO Inria-Saclay, Laboratoire des Signaux et Systèmes (L2S, UMR CNRS 8506), CNRS, CentraleSupélec, Université Paris-Sud, 3 rue Joliot Curie, 91192, Gif-sur-Yvette, France. frederic.mazencel2s. centralesupelec.fr.

Malisoff is with the Department of Mathematics, 303 Lockett Hall, Louisiana State University (LSU), Baton Rouge, LA 70803-4918, USA. malisoff@lsu.edu. Supported by NSF-ECCS Grant 1408295, and by the Roy Paul Daniels Professorship \#3 in the LSU College of Science. functions for undelayed systems into Lyapunov-Krasovskii functionals for the corresponding input delayed systems.

Another approach is the reduction model method, where the control is expressed implicitly as a solution of an integral equation, which can lead to challenging problems of numerically computing control values, especially when the system has uncertainty or the delay is time-varying. Prediction is another useful method, where the state values in the controls are replaced by numerical predictions of the state values. Standard prediction or reduction model methods can compensate for arbitrarily long input delays, but the distributed terms in their controls may make them harder to implement than emulation; see [2], [3], [14], and [19].

This note provides a new sequential predictors approach for the exponential stabilization of time-varying systems with time-varying pointwise input delays, and so builds on recent significant works such as [16] (which used LMI methods for time invariant linear systems to build sequential predictors) and [9] (which extended [16] by studying constant coefficient linear systems with time-varying delays, which is a smaller class of systems than the time-varying systems we consider here). It also extends our work [11], which was confined to constant delays. Since we do not use distributed terms or Lie derivatives in our control, our work is very different from the classical reduction model or prediction approaches that have been used by M. Krstic and others (as in [5] and [8]).

We use several dynamic extensions, with each one having the same dimension as the original system. Since we do not use distributed terms, our work differs from [1] and other works that use several dynamic extensions and distributed terms. Our result gives closed form expressions for the control and explicit lower bounds on the number of required dynamic extensions in terms of the time-varying delays and their derivatives. Our work is mainly a theoretical and methodological development. However, we illustrate our work using a pendulum dynamics that was also studied in [13], where we used a predictive approach that led to distributed controls, but [13] did not cover time-varying delays.

We use standard notation and definitions. Throughout the sequel, the dimensions are arbitrary, unless otherwise noted. For simplicity, we omit arguments of functions when they are clear from the context, and we always assume that the initial times $t_{0}$ for our solutions of our time-varying systems are $t_{0}=0$, but we can write analogs of our results for general choices of $t_{0} \geq 0$. We use $|\cdot|$ to denote the usual Euclidean norm and the induced matrix norm, and $|\phi|_{\infty}$ (resp., $|\phi|_{\mathscr{I}}$ ) denotes the essential supremum (resp., supremum over any interval $\mathscr{I}$ ) for bounded measurable functions $\phi$. 


\section{MAIN RESUlT}

We study systems of the form

$$
\dot{x}(t)=A(t) x(t)+B(t) u(t-h(t)),
$$

where the state $x$ and the control $u$ are valued in $\mathbb{R}^{n}$ and $\mathbb{R}^{\ell}$, respectively, and $h$ is a time-varying delay. Throughout this section, we make two assumptions:

Assumption 1: The function $h: \mathbb{R} \rightarrow[0, \infty)$ is $C^{1}$ and bounded from above by a constant $c_{h}>0$. Also, its derivative $\dot{h}$ is bounded from below, and $\dot{h}$ is bounded from above by a constant $l_{h} \in(0,1)$, and $\dot{h}$ has a global Lipschitz constant $n_{h}>0$.

Assumption 2: The functions $A$ and $B$ are bounded and continuous, and there is a known bounded continuous function $K:[0, \infty) \rightarrow \mathbb{R}^{\ell \times n}$ such that

$$
\dot{x}(t)=[A(t)+B(t) K(t)] x(t)
$$

is exponentially stable to 0 .

We also assume that the initial functions are constant on $\left[-c_{h}, 0\right]$. In terms of the known delay $h(t)$ and an integer $m>1$ that we specify later, we then introduce the functions

$$
\Omega_{i}(t)=t-\frac{i}{m} h(t) \text { and } \theta_{i}(t)=\Omega_{m-i+1}^{-1}\left(\Omega_{m-i}(t)\right)
$$

for all $i \in\{0, \ldots, m\}$, and we inductively define the functions

$$
R_{1}(t)=\dot{\theta}_{1}(t) \text { and } R_{i}(t)=\dot{\theta}_{i}(t) R_{i-1}\left(\theta_{i}(t)\right) \text { for } i>1 \text {. }
$$

Such functions exist by Assumption 1, which implies that the $\Omega_{i}$ 's are strictly increasing and that their ranges are all of $\mathbb{R}$. The required inverses in (3) can be computed in practice using standard programs. In terms of the positive constants

$$
u_{c}=n_{h} \frac{c_{h}}{\left(1-l_{h}\right)^{2}}+\frac{l_{h}}{1-l_{h}}
$$

and

$$
\begin{aligned}
& b_{1}=\left[1+\left(1+\frac{u_{c}}{m}\right)^{m}|A|_{\infty}\right]\left(1+\frac{u_{c}}{m}\right)^{m}|A|_{\infty}, \\
& b_{2}=\left[1+\left(1+\frac{u_{c}}{m}\right)^{m}|A|_{\infty}\right]^{2}, \text { and } b_{3}=b_{2}\left(1+\frac{u_{c}}{m}\right),
\end{aligned}
$$

and with $I_{n} \in \mathbb{R}^{n \times n}$ denoting the identity matrix, we then prove:

Theorem 1: If Assumptions 1-2 hold and $m \in \mathbb{N}$ satisfies

$$
\max \left\{2,4\left(\frac{b_{1}}{\sqrt{2}}+b_{3}\right) \frac{c_{h}}{1-l_{h}}\right\}<m
$$

then we can find positive constants $\mu_{1}$ and $\mu_{2}$ such that for all solutions of (1) in closed loop with the control

$$
u(t)=K(t+h(t)) z_{m}(t),
$$

where $z_{m}$ is the last $n$ components of the system

$$
\begin{aligned}
\dot{z}_{1}(t)= & R_{1}(t) A\left(\theta_{1}(t)\right) z_{1}(t)+R_{1}(t) B\left(\theta_{1}(t)\right) u\left(\Omega_{m-1}(t)\right) \\
& +L_{1}(t)\left[z_{1}\left(\theta_{1}^{-1}(t)\right)-x(t)\right] \\
\dot{z}_{i}(t)= & R_{i}(t) A\left(G_{i}(t)\right) z_{i}(t)+R_{i}(t) B\left(G_{i}(t)\right) u\left(\Omega_{m-i}(t)\right) \\
& +L_{i}(t)\left[z_{i}\left(\theta_{i}^{-1}(t)\right)-z_{i-1}(t)\right], i \in\{2, \ldots, m\}
\end{aligned}
$$

with the choices

$$
L_{i}(t)=-I_{n}-R_{i}(t) A\left(G_{i}(t)\right) \text { and } G_{i}=\Omega_{m}^{-1} \circ \Omega_{m-i},
$$

we have

$$
|(x(t), \mathscr{E}(t))| \leq \mu_{1}|(x, \mathscr{E})|_{\left[-c_{h}, 0\right]} e^{-\mu_{2} t}
$$

for all $t \geq 0$, where the vector $\mathscr{E}$ is defined by $\mathscr{E}(t)=\left(z_{1}(t)-\right.$ $\left.x\left(\theta_{1}(t)\right), z_{2}(t)-z_{1}\left(\theta_{2}(t)\right), \ldots, z_{m}(t)-z_{m-1}\left(\theta_{m}(t)\right)\right)$.

Remark 1: The estimate (11) means that the combined $(x, \mathscr{E})$ dynamics are uniformly globally exponentially stable to 0 . Three key features of the preceding construction are (a) our allowing arbitrarily large delay bounds $c_{h}>0$, (b) the fact that our control (8) has no distributed terms and no Lie derivatives, and (c) the fact that only the bottom $n$ components of the dynamic extension (9) are needed to compute the control values (8).

\section{SKetCh OF PROOF OF TheOREM 1}

We use this lemma, which we prove in the appendix:

Lemma 1: The $\theta_{i}$ 's in (3) and the constant $u_{c}$ in (5) satisfy

$$
\left|\dot{\theta}_{i}(t)-1\right| \leq \frac{u_{c}}{m} \text { and }\left|\theta_{i}^{-1}(t)-t\right| \leq \frac{c_{h}}{1-l_{h}} \frac{1}{m}
$$

for all $t \geq 0$ and $i \in\{1,2, \ldots, m\}$.

By Lemma 1 and our definition of the $R_{i}$ 's in (4), we get

$$
\left|R_{i}(t)\right| \leq\left(1+\frac{u_{c}}{m}\right)^{m}
$$

for all $i$, where throughout the proof, all inequalities and inequalities are for all $t \geq 0$, unless otherwise indicated. In terms of the states $z_{i}$ of (9), we define $\xi_{i}(t)=z_{i-1}\left(\theta_{i}(t)\right)$ for $i \geq 1$, where $z_{0}=x$. The rest of the proof has four parts.

Part 1: Error Dynamics. We derive a useful formula for the dynamics of the error $\mathscr{E}=\left(\mathscr{E}_{1}, \ldots, \mathscr{E}_{m}\right)$, where

$$
\mathscr{E}_{i}(t)=z_{i}(t)-\xi_{i}(t)
$$

for all $i \in\{1, \ldots, m\}$, so $\mathscr{E}_{i}(t)=z_{i}(t)-z_{i-1}\left(\theta_{i}(t)\right)$ for all $i \in$ $\{1, \ldots, m\}$. Our formulas (3) for the $\Omega_{i}$ 's and $\theta_{i}$ 's give

$$
\begin{aligned}
\dot{\xi}_{1}(t)= & R_{1}(t) A\left(\theta_{1}(t)\right) \xi_{1}(t) \\
& +R_{1}(t) B\left(\theta_{1}(t)\right) u\left(\Omega_{m-1}(t)\right) .
\end{aligned}
$$

Hence, (14) and our choice of the $z_{1}$ dynamics in (9) give

$$
\dot{\mathscr{E}}_{1}(t)=R_{1}(t) A\left(\theta_{1}(t)\right) \mathscr{E}_{1}(t)+L_{1}(t) \mathscr{E}_{1}\left(\theta_{1}^{-1}(t)\right) .
$$

For $i>1$, we can use (10) to get $G_{i-1} \circ \theta_{i}=G_{i}$, so (4) gives

$$
\begin{aligned}
\dot{\xi}_{i}(t)= & \dot{\theta}_{i}(t)\left\{R _ { i - 1 } ( \theta _ { i } ( t ) ) \left[A\left(G_{i}(t)\right) z_{i-1}\left(\theta_{i}(t)\right)\right.\right. \\
& \left.+B\left(G_{i}(t)\right) u\left(\Omega_{m-i+1}\left(\theta_{i}(t)\right)\right)\right] \\
& +L_{i-1}\left(\theta_{i}(t)\right)\left[z_{i-1}\left(\theta_{i-1}^{-1}\left(\theta_{i}(t)\right)\right)\right. \\
& \left.\left.-z_{i-2}\left(\theta_{i}(t)\right)\right]\right\} \\
= & R_{i}(t) B\left(G_{i}(t)\right) u\left(\Omega_{m-i+1}\left(\theta_{i}(t)\right)\right) \\
& +\dot{\theta}_{i}(t) L_{i-1}\left(\theta_{i}(t)\right)\left[z_{i-1}\left(\theta_{i-1}^{-1}\left(\theta_{i}(t)\right)\right)\right. \\
& \left.-z_{i-2}\left(\theta_{i}(t)\right)\right]+R_{i}(t) A\left(G_{i}(t)\right) \xi_{i}(t) .
\end{aligned}
$$

Since $\Omega_{m-i+1}\left(\theta_{i}(t)\right)=\Omega_{m-i}(t)$, we deduce that for $i \geq 2$,

$$
\begin{aligned}
\dot{\mathscr{E}}_{i}(t)= & L_{i}(t)\left[z_{i}\left(\theta_{i}^{-1}(t)\right)-z_{i-1}(t)\right] \\
& -\dot{\theta}_{i}(t) L_{i-1}\left(\theta_{i}(t)\right)\left[z_{i-1}\left(\theta_{i-1}^{-1}\left(\theta_{i}(t)\right)\right)\right. \\
& \left.-z_{i-2}\left(\theta_{i}(t)\right)\right]+R_{i}(t) A\left(G_{i}(t)\right) \mathscr{E}_{i}(t) \\
= & R_{i}(t) A\left(G_{i}(t)\right) \mathscr{E}_{i}(t)+L_{i}(t) \mathscr{E}_{i}\left(\theta_{i}^{-1}(t)\right) \\
& -\dot{\theta}_{i}(t) L_{i-1}\left(\theta_{i}(t)\right) \mathscr{E}_{i-1}\left(\theta_{i-1}^{-1}\left(\theta_{i}(t)\right)\right) .
\end{aligned}
$$

To summarize, we have the following error dynamics:

$$
\left\{\begin{aligned}
\dot{\mathscr{E}}_{1}(t)= & R_{1}(t) A\left(G_{1}(t)\right) \mathscr{E}_{1}(t)+L_{1}(t) \mathscr{E}_{1}\left(\theta_{1}^{-1}(t)\right) \\
\dot{\mathscr{E}}_{i}(t)= & R_{i}(t) A\left(G_{i}(t)\right) \mathscr{E}_{i}(t)+L_{i}(t) \mathscr{E}_{i}\left(\theta_{i}^{-1}(t)\right) \\
& -\dot{\theta}_{i}(t) L_{i-1}\left(\theta_{i}(t)\right) \mathscr{E}_{i-1}\left(\theta_{i-1}^{-1}\left(\theta_{i}(t)\right)\right) \\
& \text { for } i=2, \ldots, m
\end{aligned}\right.
$$

Part 2: Fundamental System. Motivated by the triangular structure of (18), we next study stability properties of the fundamental system

$$
\begin{aligned}
& \dot{s}_{i}(t)=R_{i}(t) A\left(G_{i}(t)\right) s_{i}(t)+L_{i}(t) s_{i}\left(\theta_{i}^{-1}(t)\right) \\
& =-s_{i}(t)+\left[I_{n}+R_{i}(t) A\left(G_{i}(t)\right)\right]\left[s_{i}(t)-s_{i}\left(\theta_{i}^{-1}(t)\right)\right]
\end{aligned}
$$


for a fixed $i$. Observe that $\theta_{i}^{-1}(t) \leq t$ for all $t \geq 0$, by our choices (3) of the $\Omega_{i}$ 's. Since $\theta_{i}^{-1}(t) \geq 0$ for all $t \geq c_{h}$, it follows that

$$
\begin{aligned}
& \dot{s}_{i}(t)=-s_{i}(t)-L_{i}(t) \int_{\theta_{i}^{-1}(t)}^{t} R_{i}(l) A\left(G_{i}(l)\right) s_{i}(l) \mathrm{d} l \\
& +L_{i}(t) \int_{\theta_{i}^{-1}(t)}^{t}\left(I_{n}+R_{i}(l) A\left(G_{i}(l)\right)\right) s_{i}\left(\theta_{i}^{-1}(l)\right) \mathrm{d} l
\end{aligned}
$$

for all $t \geq c_{h}$. We will use the function $Q\left(s_{i}\right)=\left|s_{i}\right|^{2} / 2$.

By our choices (6) of the constants $b_{1}$ and $b_{2}$, our first bound in (12) from Lemma 1, and (13), it follows that the time derivative of $Q$ along all trajectories of (20) satisfies

$$
\begin{aligned}
\dot{Q}(t) \leq & -\left|s_{i}(t)\right|^{2}+b_{1}\left|s_{i}(t)\right| \int_{t-\frac{c_{h}}{1-l_{h}} \frac{1}{m}}^{t}\left|s_{i}(l)\right| \mathrm{d} l \\
& +b_{2}\left|s_{i}(t)\right| \int_{t-\frac{c_{h}}{1-l_{h}} \frac{1}{m}}^{t}\left|s_{i}\left(\theta_{i}^{-1}(l)\right)\right| \mathrm{d} l \\
\leq & -\left|s_{i}(t)\right|^{2}+b_{1}\left|s_{i}(t)\right| \int_{t-\frac{c_{h}}{1-l_{h}} \frac{1}{m}}^{t}\left|s_{i}(l)\right| \mathrm{d} l \\
& +b_{2}\left|s_{i}(t)\right| \int_{\theta_{i}^{-1}\left(t-\frac{c_{h}}{1-l_{h}} \frac{1}{m}\right)}^{\theta_{i}^{-1}(t)}\left|s_{i}(r)\right|\left|\dot{\theta}_{i}(r)\right| \mathrm{d} r .
\end{aligned}
$$

Here and in what follows, all inequalities and equalities are for all $t \geq \max \left\{c_{h}, 2 c_{h} /\left(m\left(1-l_{h}\right)\right)\right\}$, unless otherwise indicated. Hence, since $\theta_{i}^{-1}(t) \leq t$ for all $t \geq 0$, (12) gives

$$
\begin{aligned}
\dot{Q}(t) \leq & -\left|s_{i}(t)\right|^{2}+b_{1}\left|s_{i}(t)\right| \int_{t-\frac{c_{h}}{1-l_{h}} \frac{1}{m}}^{t}\left|s_{i}(l)\right| \mathrm{d} l \\
& +b_{3}\left|s_{i}(t)\right| \int_{t-\frac{2 c_{h}}{1-l_{h}} \frac{1}{m}}^{t}\left|s_{i}(r)\right| \mathrm{d} r .
\end{aligned}
$$

Since (7) gives

$$
\frac{2 c_{h}}{m\left(1-l_{h}\right)}<\frac{1}{b_{1} / \sqrt{2}+b_{3}},
$$

we can use Young's inequality twice (i.e., $s_{i}(t) r \leq \frac{1}{4}\left|s_{i}(t)\right|^{2}+$ $r^{2}$ for suitable $r \geq 0$ ) and then Jensen's inequality (applied to the convex integrand function $\mathscr{M}(s)=|s|^{2}$ ) to get

$$
\begin{aligned}
\dot{Q}(t) \leq & -\frac{1}{2}\left|s_{i}(t)\right|^{2}+\frac{\left(b_{1} / \sqrt{2}\right)^{2}}{b_{1} / \sqrt{2}+b_{3}} \int_{t-\frac{c_{h}}{1-l_{h} \frac{1}{m}}}^{t}\left|s_{i}(l)\right|^{2} \mathrm{~d} l \\
& +\frac{b_{3}^{2}}{b_{1} / \sqrt{2}+b_{3}} \int_{t-\frac{2 c_{h}}{1-l_{h}} \frac{1}{m}}^{t}\left|s_{i}(r)\right|^{2} \mathrm{~d} r \\
\leq & -\frac{1}{2}\left|s_{i}(t)\right|^{2}+\left(\frac{b_{1}}{\sqrt{2}}+b_{3}\right) \int_{t-\frac{2 c_{h}}{1-l_{h}} \frac{1}{m}}^{t}\left|s_{i}(r)\right|^{2} \mathrm{~d} r
\end{aligned}
$$

where the first application of Young's and Jensen's inequalities that we used to obtain the first inequality in (24) was

$$
\begin{aligned}
& b_{1}\left|s_{i}(t)\right| \int_{t-\frac{c_{h}}{1-l_{h}} \frac{1}{m}}^{t}\left|s_{i}(l)\right| \mathrm{d} l \\
& \leq \frac{1}{4}\left|s_{i}(t)\right|^{2}+\left(b_{1} \int_{t-\frac{c_{h}}{1-l_{h}} \frac{1}{m}}^{t}\left|s_{i}(l)\right| \mathrm{d} l\right)^{2} \\
& \leq \frac{1}{4}\left|s_{i}(t)\right|^{2}+b_{1}^{2} \frac{c_{h}}{1-l_{h}} \frac{1}{m} \int_{t-\frac{c_{h}}{1-l_{h}} \frac{1}{m}}^{t}\left|s_{i}(l)\right|^{2} \mathrm{~d} l \\
& \leq \frac{1}{4}\left|s_{i}(t)\right|^{2}+\frac{\left(b_{1} / \sqrt{2}\right)^{2}}{b_{1} / \sqrt{2}+b_{3}} \int_{t-\frac{c_{h}}{1-l_{h}} \frac{1}{m}}^{t}\left|s_{i}(l)\right|^{2} \mathrm{~d} l,
\end{aligned}
$$

and the second one was analogous, and then we used the fact that $\left(r^{2}+s^{2}\right) /(r+s) \leq r+s$ for all $r \geq 0$ and $s>0$, with the choices $r=b_{1} / \sqrt{2}$ and $s=b_{3}$.
Since our lower bound on $m$ in (7) also allows us to find a constant $\lambda>1$ that is close enough to 1 so that

$$
4\left(\frac{b_{1}}{\sqrt{2}}+b_{3}\right) \frac{\lambda c_{h}}{1-l_{h}}<m
$$

it follows from (24) that we can also find a constant $c_{0}>0$ such that the time derivative of

$$
Q^{\sharp}\left(s_{i, t}\right)=Q\left(s_{i}(t)\right)+\lambda\left(\frac{b_{1}}{\sqrt{2}}+b_{3}\right) \int_{t-\frac{2 c_{h}}{1-l_{h}} \frac{1}{m}}^{t} \int_{w}^{t}\left|s_{i}(r)\right|^{2} \mathrm{~d} r \mathrm{~d} w
$$

along all solutions of the fundamental system (19) satisfies

$$
\begin{aligned}
\dot{Q}^{\sharp} \leq & -\frac{1}{2}\left|s_{i}(t)\right|^{2}+\left(\frac{b_{1}}{\sqrt{2}}+b_{3}\right) \frac{2 c_{h}}{1-l_{h}} \frac{\lambda}{m}\left|s_{i}(t)\right|^{2} \\
& +(1-\lambda)\left(\frac{b_{1}}{\sqrt{2}}+b_{3}\right) \int_{t-\frac{2 c_{h}}{m\left(1-l_{h}\right)}}^{t}\left|s_{i}(r)\right|^{2} \mathrm{~d} r \\
\leq & -c_{0} Q^{\sharp}\left(s_{i, t}\right),
\end{aligned}
$$

where the second inequality in (27) used the bound

$$
\int_{t-\frac{2 c_{h}}{1-l} \frac{1}{m}}^{t} \int_{w}^{t}\left|s_{i}(r)\right|^{2} \mathrm{~d} r \mathrm{~d} w \leq \frac{2 c_{h}}{\left(1-l_{h}\right) m} \int_{t-\frac{2 c_{h}}{1-l_{h}} \frac{1}{m}}^{t}\left|s_{i}(r)\right|^{2} \mathrm{~d} r
$$

and $s_{i, t}(\ell)=s_{i}(t+\ell)$ for all $\ell \in\left[-2 c_{h} /\left(\left(1-l_{h}\right) m\right), 0\right]$. This concludes our analysis of the fundamental system (19).

Part 3: Exponential Stability of the Error Dynamics (18) This part entails finding positive constants $\omega_{i}>0$ and $\eta_{i}>0$ such that along all solutions of the dynamics for

$$
\mathscr{E}^{\sharp}(t)=\left(\mathscr{E}_{1}\left(\theta_{1}^{-1} \circ \theta_{m}(t)\right), \mathscr{E}_{2}\left(\theta_{2}^{-1} \circ \theta_{m}(t)\right), \ldots, \mathscr{E}_{m}(t)\right),
$$

the function

$Q_{m}^{\sharp}\left(\mathscr{E}_{t}^{\sharp}\right)=\omega_{1} Q^{\sharp}\left(\mathscr{E}_{1, t}^{\sharp}\right)+\ldots+\omega_{m-1} Q^{\sharp}\left(\mathscr{E}_{m-1, t}^{\sharp}\right)+\omega_{m} Q^{\sharp}\left(\mathscr{E}_{m, t}^{\sharp}\right)$

satisfies

$$
\dot{Q}_{m}^{\sharp}\left(\mathscr{E}_{t}^{\sharp}\right) \leq-\eta_{1} Q_{m}^{\sharp}\left(\mathscr{E}_{t}^{\sharp}\right) .
$$

This can be done by an inductive argument, using our lower and upper bounds on $\dot{h}$ from Assumption 1. For instance, we can use the triangle inequality to prove that along all solutions for the $\left(\mathscr{E}_{1}^{\sharp}(t), \mathscr{E}_{2}^{\sharp}(t)\right)$ dynamics, we have

$$
\begin{aligned}
& \frac{d}{d t} Q^{\sharp}\left(\mathscr{E}_{2, t}^{\sharp}\right) \leq-\frac{\underline{\theta} c_{0}}{2} Q^{\sharp}\left(\mathscr{E}_{2, t}^{\sharp}\right)+\frac{2 \bar{\theta}^{2}}{\underline{\theta} c_{0}}\left|\dot{\theta}_{2}\right|_{\infty}^{2}\left|L_{1}\right|_{\infty}^{2} Q^{\sharp}\left(\mathscr{E}_{1, t}^{\sharp}\right) \\
& \frac{d}{d t} Q^{\sharp}\left(\mathscr{E}_{1, t}^{\sharp}\right) \leq-\underline{\theta} c_{0} Q^{\sharp}\left(\mathscr{E}_{1, t}^{\sharp}\right),
\end{aligned}
$$

where the positive constants $\underline{\theta}$ and $\bar{\theta}$ are chosen such that $\underline{\theta} \leq\left(\theta_{i}^{-1} \circ \theta_{m}\right)^{\prime}(t) \leq \bar{\theta}$ for all $t \geq 0$ and all $i \in\{1,2, \ldots, m\}$. Hence, the function

$$
Q_{2}^{\sharp}\left(\mathscr{E}_{1, t}^{\sharp}, \mathscr{E}_{2, t}^{\sharp}\right)=Q^{\sharp}\left(\mathscr{E}_{2, t}^{\sharp}\right)+\left(1+2\left(\frac{\bar{\theta}\left|\dot{\theta}_{2}\right|_{\infty}\left|L_{1}\right|_{\infty}}{\underline{\theta} c_{0}}\right)^{2}\right) Q^{\sharp}\left(\mathscr{E}_{1, t}^{\sharp}\right)
$$

satisfies

$$
\dot{Q}_{2}^{\sharp} \leq-\frac{\underline{\theta} c_{0}}{2}\left(Q^{\sharp}\left(\mathscr{E}_{1, t}^{\sharp}\right)+Q^{\sharp}\left(\mathscr{E}_{2, t}^{\sharp}\right)\right),
$$

and so is a Lyapunov-Krasovskii functional for the $\left(\mathscr{E}_{1}^{\sharp}(t), \mathscr{E}_{2}^{\sharp}(t)\right)$ dynamics, and this process can be continued.

Part 4: Exponential Stability of (1) in Closed Loop with (8). By our error variable formulas (14) and $\mathscr{E}_{i}(t)=z_{i}(t)-$ $z_{i-1}\left(\theta_{i}(t)\right)$ for all $i \in\{1, \ldots, m\}$, we have

$$
\begin{aligned}
& z_{m}(t)=\xi_{m}(t)+\mathscr{E}_{m}(t)=z_{m-1}\left(\theta_{m}(t)\right)+\mathscr{E}_{m}(t) \\
& =z_{m-2}\left(\theta_{m-1}\left(\theta_{m}(t)\right)\right)+\mathscr{E}_{m-1}\left(\theta_{m}(t)\right)+\mathscr{E}_{m}(t),
\end{aligned}
$$

and therefore we can argue inductively to get

$$
\begin{aligned}
& z_{m}(t)=x\left(\theta_{1}\left(\ldots \theta_{m-1}\left(\theta_{m}(t)\right) \ldots\right)\right) \\
& +\mathscr{E}_{1}\left(\theta_{2}\left(\ldots \theta_{m}(t) \ldots\right)\right)+\ldots+\mathscr{E}_{m}(t) .
\end{aligned}
$$


Then our formulas for the $\theta_{i}$ 's give

$$
z_{m}(t)=x\left(\Omega_{m}^{-1}(t)\right)+\mathscr{E}_{1}\left(\theta_{2}\left(\ldots \theta_{m}(t) \ldots\right)\right)+\ldots+\mathscr{E}_{m}(t) .
$$

Hence, our control (8) can be written as

$$
\begin{aligned}
& u(t-h(t))=K(t)\left[x\left(\Omega_{m}^{-1}(t-h(t))\right)\right. \\
& \left.+\mathscr{E}_{1}\left(\theta_{2}\left(\ldots \theta_{m}(t-h(t) \ldots)\right)\right)+\ldots+\mathscr{E}_{m}(t-h(t))\right] .
\end{aligned}
$$

Since $\Omega_{m}(t)=t-h(t)$, the closed loop system (1) is then

$$
\begin{aligned}
& \dot{x}(t)=(A(t)+B(t) K(t)) x(t) \\
& +\left\{B ( t ) \left[K(t) \mathscr{E}_{1}\left(\theta_{2}\left(\ldots \theta_{m}(t-h(t) \ldots)\right)\right)\right.\right. \\
& \left.\left.+\ldots+K(t) \mathscr{E}_{m}(t-h(t))\right]\right\} .
\end{aligned}
$$

Also, Assumption 2 provides a $C^{1}$ bounded function $P$ such that the Lyapunov function $V(t, x)=x^{\top} P(t) x$ has a quadratic lower bound in $|x|$ and satisfies $\dot{V} \leq-|x(t)|^{2}$ along all solutions of (2) (using [7, Theorem 4.14]). Then the triangle inequality gives

$$
\begin{aligned}
\dot{V} \leq & -\frac{1}{2}|x(t)|^{2}+2|P|_{\infty}^{2}\left|\delta^{\sharp}\right|_{\infty}^{2} \\
\leq & -\frac{1}{2|P|_{\infty}} V(t, x(t))+2|P|_{\infty}^{2}\left|\delta^{\sharp}\right|_{\infty}^{2} \\
\leq & -\frac{1}{2|P|_{\infty}} V(t, x(t)) \\
& +2 m\left(|P|_{\infty}|B|_{\infty}|K|_{\infty}\right)^{2} \\
& \times\left(\mid \mathscr{E}_{1}\left(\left.\theta_{2}\left(\ldots \theta_{m}(t-h(t) \ldots)\right)\right|^{2}+\ldots\right.\right. \\
& \left.+\left|\mathscr{E}_{m}(t-h(t))\right|^{2}\right)
\end{aligned}
$$

along all solutions of (35), where $\delta^{\sharp}$ is the quantity in curly braces in (35). One then can show that the function

$$
\begin{aligned}
& Q_{+}^{\sharp}\left(\mathscr{E}_{t}^{\sharp \sharp}\right)=V(t, x(t)) \\
& +\left(1+\frac{2 m}{\underline{\underline{M}} \eta_{1}}\left(|P|_{\infty}|B|_{\infty}|K|_{\infty}\right)^{2}\right) \sum_{i=1}^{m} \frac{2}{\omega_{i}} Q_{m}^{\sharp}\left(\mathscr{E}_{\mathscr{M}_{i}(t)}^{\sharp}\right)
\end{aligned}
$$

admits a constant $d_{0}>0$ such that the Lyapunov-Krasovskii decay condition

$$
\dot{Q}_{+}^{\sharp}\left(\mathscr{E}_{t}^{\sharp \sharp}\right) \leq-d_{0} Q_{+}^{\sharp}\left(\mathscr{E}_{t}^{\sharp \sharp}\right)
$$

holds along all solutions of the dynamics for

$$
\mathscr{E}^{\mathscr{\sharp} \sharp}(t)=\left(x(t), \mathscr{E}_{\mathscr{M}_{1}(t)}^{\sharp}, \ldots, \mathscr{E}_{\mathscr{M}_{m}(t)}^{\sharp}\right),
$$

where $\mathscr{M}_{i}(t)=\theta_{m}^{-1} \circ \theta_{i} \circ \theta_{i+1} \circ \ldots \circ \theta_{m}(t-h(t))=\Omega_{1} \circ$ $\Omega_{m-i+1}^{-1} \circ \Omega_{m}(t)$ for $i=1,2, \ldots, m-1$ and $\mathscr{M}_{m}(t)=t-h(t)=$ $\Omega_{m}(t)$, by choosing positive constants $\mathscr{M}$ and $\overline{\mathscr{M}}$ such that $\underline{\mathscr{M}} \leq \dot{\mathscr{M}}_{i}(t) \leq \overline{\mathscr{M}}$ for all $i \in\{1,2, \ldots, m\}$ and $t \geq 0$. The final uniform global exponential stability estimate for the $(x, \mathscr{E})$ dynamics follows from the quadratic structure of $V$ and $Q$, the invertibility of the functions $\mathscr{H}_{i}=\theta_{i}^{-1} \circ \theta_{m} \circ \mathscr{M}_{i}$ for all $i$, the existence of positive constants $h_{a}$ and $h_{b}$ such that $\mathscr{H}_{i}^{-1}(t) \geq h_{a} t-h_{b}$ for all $i$ and $t \geq 0$ (which follows from our bounds on $\dot{h}$ from Assumption 1 and the Mean Value Theorem), and the subadditivity of the square root.

Remark 2: Since $\Omega_{m}(t)=t-h(t)$, our control is

$$
\begin{aligned}
& u(t-h(t))=K(t) x(t)+ \\
& K(t)\left[\mathscr{E}_{1}\left(\theta_{2}\left(\ldots \theta_{m}(t-h(t) \ldots)\right)\right)+\ldots+\mathscr{E}_{m}(t-h(t))\right],
\end{aligned}
$$

which may at first seem to contradict our assumption that the current value of the state $x(t)$ is not available to use in the control. However, our formulas (3) for the $\theta_{i}$ 's imply that $\theta_{2} \circ \ldots \circ \theta_{m}(t-h(t))=\theta_{1}^{-1}(t)$ for all $t \geq 0$. This gives

$$
\begin{aligned}
\mathscr{E}_{1}\left(\theta_{2}\left(\ldots \theta_{m}(t-h(t) \ldots)\right)\right) & =z_{1}\left(\theta_{1}^{-1}(t)\right)-\xi_{1}\left(\theta_{1}^{-1}(t)\right) \\
& =z_{1}\left(\theta_{1}^{-1}(t)\right)-x(t),
\end{aligned}
$$

so two terms in (40) containing $x(t)$ cancel. Hence, our control does not require current values $x(t)$.

\section{ApplicAtion to PENDUlum}

Consider the model

$$
\left\{\begin{array}{l}
\dot{r}_{1}(t)=r_{2}(t) \\
\dot{r}_{2}(t)=-\frac{g}{l} \sin \left(r_{1}(t)\right)+\frac{1}{\overline{\bar{m}} l^{2}} v(t-h(t))
\end{array}\right.
$$

of the simple pendulum with a time-varying delay $h(t)$, where $g=9.8 \mathrm{~m} / \mathrm{s}$ is the gravity constant, $l$ is the pendulum length in meters, $\bar{m}$ is the pendulum mass, and $v$ is the input. As in [11] and [13], our control objective is to track a given $C^{1}$ reference trajectory $\left(r_{1, s}(t), r_{2, s}(t)\right)$ such that $\dot{r}_{1, s}(t)=r_{2, s}(t)$. Using the error variables $\tilde{r}_{i}=r_{i}-r_{i, s}(t)$ for $i=1,2$ and the change of feedback

$$
u(t-h(t))=\frac{1}{\overline{\bar{m}} l^{2}} v(t-h(t))-\dot{r}_{2, s}(t)-\frac{g}{l} \sin \left(r_{1, s}(t)\right),
$$

we obtain the tracking system

$$
\left\{\begin{aligned}
\dot{\tilde{r}}_{1}(t)= & \tilde{r}_{2}(t) \\
\dot{\tilde{r}}_{2}(t)= & \frac{g}{l}\left[\sin \left(r_{1, s}(t)\right)-\sin \left(\tilde{r}_{1}(t)+r_{1, s}(t)\right)\right] \\
& +u(t-h(t)) .
\end{aligned}\right.
$$

The work [13] showed that when $r_{1, s}(t)=\omega t$ and $\omega>0$ is a large enough constant and $h=1$, the linearization

$$
\left\{\begin{array}{l}
\dot{x}_{1}(t)=x_{2}(t) \\
\dot{x}_{2}(t)=-\frac{g}{l} \cos (\omega t) x_{1}(t)+u(t-h)
\end{array}\right.
$$

of (44) at 0 has the globally exponentially stabilizing distributed control $u$ defined by

$$
\begin{aligned}
& u(t)=-0.6 x_{1}(t)-0.4 x_{2}(t) \\
& -\int_{t-1}^{t}(0.6(t-r-1)+0.4) u(r) \mathrm{d} r .
\end{aligned}
$$

Our work [11] designed a globally asymptotically stabilizing sequential predictor control for the original nonlinear system (44) for cases where $h$ is constant. However, it is not obvious how to extend the analysis from [11] to time-varying delays. Therefore, we apply Theorem 1 to its linearization (45) using the time-varying delay $h(t)=1+\alpha \sin (t)$, where $\alpha \in(0,1)$ is a known constant, but analogous reasoning applies for any delay $h(t)$ satisfying Assumption 1 above.

To this end, first note that for any choice of the constant $\omega>0$, Assumption 2 is satisfied using

$$
\begin{aligned}
& A(t)=\left[\begin{array}{cc}
0 & 1 \\
-\frac{g}{l} \cos (\omega t) & 0
\end{array}\right], B=\left[\begin{array}{l}
0 \\
1
\end{array}\right], \\
& \text { and } K(t)=\left[\frac{g}{l} \cos (\omega t)-1 \quad-1\right]
\end{aligned}
$$

and then $|A|_{\infty}=\max \{g / l, 1\}$. Therefore, our requirement (7) on $m \geq 2$ from Theorem 1 holds if

$$
\begin{aligned}
& \frac{m}{4}-\frac{1+\alpha}{1-\alpha}\left[\frac{\max \{g / l, 1\}}{\sqrt{2}}\left(1+\max \left\{\frac{g}{l}, 1\right\}\left(1+\frac{u_{c}}{m}\right)^{m}\right)\left(1+\frac{u_{c}}{m}\right)^{m}\right. \\
& \left.+\left(1+\max \left\{\frac{g}{l}, 1\right\}\left(1+\frac{u_{c}}{m}\right)^{m}\right)^{2}\left(1+\frac{u_{c}}{m}\right)\right]>0 .
\end{aligned}
$$

Condition (48) can easily be checked for suitable choices of $\alpha$, and $l$. For instance, if we pick $\alpha=1 / 7$, any $\omega>0$, any $l>g$, and the choice $m=47$, then we get the value 0.376057 for the left side of (48), so Theorem 1 applies with $m=47$. 


\section{CONClusions ANd Future Work}

We designed uniformly globally exponentially stabilizing sequential predictor controllers for a large class of timevarying linear systems under general conditions on the timevarying input delays. By allowing arbitrarily large bounds on the input delays, we overcame a possible challenge in applying emulation methods to systems that can have long input delays. We provided a formula for finding the required numbers of sequential predictors that are needed to realize our stability objectives.

We hope to extend our work to systems with uncertainties, and to adaptive systems, where dynamic extensions identify unknown parameters. We also hope to cover nonlinear systems with time-varying delays, by building sequential predictors for the linear parts, and proving that the controls ensure local stabilization of the original nonlinear systems. Finally, we plan to merge our methods with the work [10], by proving robust forward invariance under state constraints and time-varying delays.

\section{APPENDIX: PROOF OF LEMMA 1}

Fix any $i \in\{1,2, \ldots, m\}$ and $t \geq 0$. Our formula $\theta_{i}(t)=$ $\Omega_{m-i+1}^{-1}\left(\Omega_{m-i}(t)\right)$ from (3) gives

$$
\begin{aligned}
\theta_{i}(t)= & t+\Omega_{m-i+1}^{-1}\left(\Omega_{m-i+1}(t)+\frac{1}{m} h(t)\right) \\
& -\Omega_{m-i+1}^{-1}\left(\Omega_{m-i+1}(t)\right) .
\end{aligned}
$$

Hence, the Mean Value Theorem provides a $w \in \mathbb{R}$ (depending on $t$ and $i$ in general) such that

$$
\theta_{i}(t)=t+\frac{1}{\Omega_{m-i+1}(w)} \frac{1}{m} h(t) .
$$

Therefore, Assumption 1 gives

$$
\left|\theta_{i}(t)-t\right| \leq \frac{1}{1-l_{h}} \frac{1}{m} h(t) \leq \frac{c_{h}}{1-l_{h}} \frac{1}{m},
$$

which gives the second inequality in (12), by the invertibility of $\theta_{i}$.

To check the first inequality in (12), first note that

$$
\begin{aligned}
\dot{\theta}_{i}(t)= & \frac{1-\frac{m-i}{m} \dot{h}(t)}{1-\frac{m-i+1}{m} \dot{h}\left(\Omega_{m-i+1}^{-1}\left(\Omega_{m-i}(t)\right)\right)} \\
= & \frac{1-\frac{m-i+1}{m} h(t)}{1-\frac{m-i+1}{m} \dot{h}\left(\Omega_{m-i+1}^{-1}\left(\Omega_{m-i}(t)\right)\right)} \\
& +\frac{\frac{1}{m} \dot{h}(t)}{1-\frac{m-i+1}{m} \dot{h}\left(\Omega_{m-i+1}^{-1}\left(\Omega_{m-i}(t)\right)\right)} .
\end{aligned}
$$

Since $\Omega_{m-i}(t)=\Omega_{m-i+1}(t)+\frac{1}{m} h(t)$, the function

$$
\mathscr{G}_{i}(t)=\Omega_{m-i+1}^{-1}\left(\Omega_{m-i+1}(t)+\frac{1}{m} h(t)\right)
$$

is such that

$$
\begin{aligned}
\dot{\theta}_{i}(t)= & \frac{1-\frac{m-i+1}{m} \dot{h}(t)}{1-\frac{m-i+1}{m} \dot{h}\left(\mathscr{G}_{i}(t)\right)}+\frac{\frac{1}{m} \dot{h}(t)}{1-\frac{m-i+1}{m} \dot{h}\left(\Omega_{m-i+1}^{-1}\left(\Omega_{m-i}(t)\right)\right)} \\
= & \frac{m-i+1}{m} \frac{\dot{h}\left(t+\mathscr{G}_{i}(t)-\Omega_{m-i+1}^{-1}\left(\Omega_{m-i+1}(t)\right)\right)-\dot{h}(t)}{1-\frac{m-i+1}{m} \dot{h}\left(\mathscr{G}_{i}(t)\right)} \\
& +\frac{1}{m} \frac{1}{1-\frac{m-i+1}{m} \dot{h}\left(\Omega_{m-i+1}^{-1}\left(\Omega_{m-i}(t)\right)\right)}+1,
\end{aligned}
$$

by writing $\dot{h}(t)$ in the first numerator in (A.6) as

$$
\dot{h}(t)=\left(\dot{h}(t)-\dot{h}\left(\mathscr{G}_{i}(t)\right)+\dot{h}\left(\mathscr{G}_{i}(t)\right) .\right.
$$

It follows that

$$
\begin{aligned}
\left|\dot{\theta}_{i}(t)-1\right| \leq & \frac{m-i+1}{m} n_{h} \frac{\left|\mathscr{S}_{i}(t)-\Omega_{m-i+1}^{-1}\left(\Omega_{m-i+1}(t)\right)\right|}{1-\frac{m-i+1}{m} l_{h}} \\
& +\frac{1}{m} \frac{l_{h}}{1-\frac{m-i+1}{m} l_{h}},
\end{aligned}
$$

using the Lipschitz constant $n_{h}$ for $\dot{h}$. Hence, our choice of $\mathscr{G}_{i}$ and the Mean Value Theorem applied to $\Omega_{m-i+1}^{-1}$ gives

$$
\begin{aligned}
\left|\dot{\theta}_{i}(t)-1\right| \leq & \frac{m-i+1}{m} n_{h} \frac{\left|\Omega_{m-i+1}(t)+\frac{1}{m} h(t)-\Omega_{m-i+1}(t)\right|}{\left(1-\frac{m-i+1}{m} l_{h}\right)^{2}} \\
& +\frac{1}{m} \frac{l_{h}}{1-\frac{m-i+1}{m} l_{h}} \\
\leq & \frac{1}{m} \frac{m-i+1}{m} n_{h} \frac{c_{h}}{\left(1-\frac{m-i+1}{m} l_{h}\right)^{2}}+\frac{1}{m} \frac{l_{h}}{1-\frac{m-i+1}{m} l_{h}} .
\end{aligned}
$$

The lemma now follows from our formula (5) for $u_{c}$.

\section{REFERENCES}

[1] T. Ahmed-Ali, I. Karafyllis, and F. Lamnabhi-Lagarrigue. Global exponential sampled-data observers for nonlinear systems with delayed measurements. Systems and Control Letters, 67(7):539-549, 2013.

[2] Z. Artstein. Linear systems with delayed controls: a reduction. IEEE Trans. Automat. Control, 27(4):869-879, 1982.

[3] N. Bekiaris-Liberis and M. Krstic. Compensation of time-varying input and state delays for nonlinear systems. Journal of Dynamic Systems, Measurement and Control, 134(1):1-14, 2012.

[4] N. Bekiaris-Liberis and M. Krstic. Nonlinear Control Under Nonconstant Delays. Society for Industrial and Applied Mathematics, Philadelphia, PA, 2013.

[5] D. Bresch-Pietri and N. Petit. Robust compensation of a chattering time-varying input delay. In Proceedings of the IEEE Conference on Decision and Control, pages 457-462, Los Angeles, LA, 2014.

[6] R. Kamalapurkar, N. Fischer, S. Obuz, and W. Dixon. Time-varying input and state delay compensation for uncertain nonlinear systems. IEEE Trans. Automat. Control, 61(3):834-839, 2016.

[7] H. Khalil. Nonlinear Systems, Third Edition. Prentice Hall, Upper Saddle River, NJ, 2002.

[8] M. Krstic. Delay Compensation for Nonlinear, Adaptive, and PDE Systems. Birkhauser, Boston, 2009.

[9] V. Léchappé. Predictive Control and Estimation of Uncertain Systems with an Input Delay. PhD Thesis, Ecole Centrale de Nantes, Nantes, France, 2015.

[10] M. Malisoff and F. Zhang. Robustness of adaptive control under time delays for three-dimensional curve tracking. SIAM Journal on Control and Optimization, 53(4):2203-2236, 2015.

[11] F. Mazenc and M. Malisoff. Stabilization of nonlinear time-varying systems through a new prediction based approach. IEEE Trans. Automat. Control, to appear. DOI: 10.1109/TAC.2016.2600500.

[12] F. Mazenc, M. Malisoff, and Z. Lin. Further results on input-to-state stability for nonlinear systems with delayed feedbacks. Automatica, 44(9):2415-2421, 2008.

[13] F. Mazenc, M. Malisoff, and S-I. Niculescu. Reduction model approach for linear time-varying systems with delays. IEEE Trans. Automat. Control, 59(8):2068-2082, 2014.

[14] F. Mazenc, S-I. Niculescu, and M. Krstic. Lyapunov-Krasovskii functionals and application to input delay compensation for linear time-invariant systems. Automatica, 48(7):1317-1323, 2012.

[15] M. Merad, R. Downey, S. Obuz, and W. Dixon. Isometric torque control for neuromuscular electrical stimulation with time-varying input delay. IEEE Trans. Control Systems Technology, 24(3):971-978, 2016.

[16] M. Najafi, S. Hosseinnia, F. Sheikholeslam, and M. Karimadin Closed-loop control of dead time systems via sequential subpredictors. International Journal of Control, 86(4):599-609, 2013.

[17] J-P. Richard. Time-delay systems: an overview of some recent advances and open problems. Automatica, 39(10):1667-1694, 2003.

[18] N. Sharma, C. Gregory, and W. Dixon. Predictor-based compensation for electromechanical delay during neuromuscular electrical stimulation. IEEE Trans. Neural Systems and Rehabilitation Engineering, 19(6):601-611, 2011.

[19] E. Witrant, C. Canudas de Wit, D. Georges, and M. Alamir. Remote stabilization via communication networks with a distributed control law. IEEE Trans. Automat. Control, 52(8):1480-1485, 2007. 